\title{
Numerical Double Integration for Unequal Data Spaces
}

\author{
Md. Nayan Dhali \\ Assistant Professor, Department of Mathematics, Jashore University of Science and Technology, Bangladesh \\ Corresponding Author: mndhali_math@just.edu.bd
}

Nandita Barman

Assistant Professor, Department of ICT, Bangladesh University of Professionals, Bangladesh

\section{Md. Mohedul Hasan}

Lecturer, Department of Mathematics, Dhaka University of Engineering and Technology, Bangladesh

\author{
A. K. M. Selim Reza \\ Lecturer, Department of Mathematics, Khulna University of Engineering and Technology, Bangladesh
}

Received: 03 October 2020; Accepted: 03 November 2020; Published: 08 December 2020

\begin{abstract}
Numerical integral is one of the mathematical branches that connect between analytical mathematics and computer. Numerical integration is a primary tool used by engineers and scientists to obtain an approximate result for definite integrals that cannot be solved analytically. Numerical double integration is widely used in calculating surface area, the intrinsic limitations of flat surfaces and finding the volume under the surface. A wide range of method is applied to solve numerical double integration for equal data space but the difficulty is arisen when the data values are not equal. In this paper we have tried to generate a mathematical formula of numerical double integration for unequal data spaces. Trapezoidal rule for unequal space is used to evaluate the formula. We also verified our proposed model by demonstrating some numerical examples and compared the numerical result with the analytical result.
\end{abstract}

Index Terms: Numerical Integral, Numerical Double Integration, Newton`s Divided Difference, Trapezoidal Rule

\section{Introduction}

Numerical integration is the method of predict the value of a definite integral from a set of numerical values of the integrand. Whenever a technical problem leads to a differential equations which cannot be integrated in closed form, approximate methods of solutions must be employed. These methods are based on series expansions or they may be purely numerical methods leading to the evaluation of the unknown integral at specified points of its interval of definitions but simple arithmetic means. Double integrals are widely used to calculate the area of a region, the volume under a surface, and the average value of a function of two variables over a rectangular region. In general, solving the two-fold integral is not easy to do analytically. Therefore, we need a numerical method to get the solution. Numerical methods can only provide solutions that approach true value. To solve numerical double integral the multiple-segment trapezoidal or Simpson's rule would be applied in the first dimension with each value of the second dimension held constant. Then the method would be applied to integrate the second dimension. A lot of works have been found to solve numerical double integration for equal data sets. But researchers have faced troubles to solve problems when the data sets are not equal. In this paper we mainly focused on formulating a mathematical formula to calculate numerical double integration for unequal data spaces. We are hopeful that our work will be effective for scientists to solve numerical double integration when the data values are unequal.

Md. Nayan Dhali, Mohammad Farhad Bulbul and Umme Sadiya have tried to establish a comparison among Trapezoidal rule, Simpson 1/3 and Simpson 3/8 rule for unequal data space and they found that Simpson's 1/3 provide better result than the others[6]. Md. Mamun-Ur -Rashid khan, M.R. Hossain and Selina Parvin developed a new approach of numerical integration schemes for unequal data space. Romesh Kumar Muthumalai tried to determine the error of numerical integration and differentiation and he also derived some formula for numerical differentiation through divided difference and these new formulas are quite useful to approximate derivatives when additional information about derivatives at some point is given. Safaa M. Aljassas developed a new numerical approach to evaluate Double integrals with Continuous Integrands [11, 12, 13]. Andika Saputra, Rizal Bakri and Ramlan Mahmud have used Romberg method to 
analyze double integration for trigonometric function [14]. Rajinder Thukral accelerated the Simpson's method for solving nonlinear equations. In this purpose he represented an improvement of the classical Simpson's third order method for finding zeros of nonlinear equations and introduced a new formula for approximating second order derivative [17].

The rest of the paper will be described as follows: In section two, we have derived a mathematical model to solve numerical double integration for unequal data sets. In section three, some numerical example be examined and verified the result with actual result and finally we draw a conclusion.

\section{Numerical Double Integration for Unequal Space}

In this section we will discuss numerical double integration for unequal space. This formula can be applied to solve double integration when the numeric sets are not equal. We can be obtained this formula by applying trapezoidal rule for unequal space that was derived in this chapter. For do this we consider a double integral is defined by

$$
I=\int_{y_{j}}^{y_{j+1}} \int_{x_{i}}^{x_{i+1}} f(x, y) d x d y
$$

Where the distance of each interval $\left[\mathrm{x}_{\mathrm{i}}, \mathrm{x}_{\mathrm{i}+1}\right]$ and $\left[\mathrm{y}_{\mathrm{j}}, \mathrm{y}_{\mathrm{j}+1}\right]$ are not equal.

From Newton`s divided difference

$\mathrm{y}=\mathrm{y}_{0}+\left(\mathrm{x}-\mathrm{x}_{0}\right)\left[\mathrm{x}_{0}, \mathrm{x}_{1}\right]+\left(\mathrm{x}-\mathrm{x}_{0}\right)\left(\mathrm{x}-\mathrm{x}_{1}\right)\left[\mathrm{x}_{0}, \mathrm{x}_{1}, \mathrm{x}_{2}\right]+\cdots$

Trapezoidal rule for the unequal space

Similarly, for the interval $\left[x_{1}, x_{2}\right]$ we deduce,

$$
\begin{aligned}
\int_{x_{0}}^{x_{1}} y d x & =\int_{x_{0}}^{x_{1}}\left(y_{0}+\left(x-x_{0}\right)\left[x_{0}, x_{1}\right]\right) d x \\
& =y_{0}\left(x_{1}-x_{0}\right)+\frac{\left(x_{1}-x_{0}\right)^{2}}{2}\left[x_{0}, x_{1}\right] \\
& =y_{0}\left(x_{1}-x_{0}\right)+\frac{\left(x_{1}-x_{0}\right)^{2}}{2}\left(\frac{y_{0}}{\left(x_{0}-x_{1}\right)}+\frac{y_{1}}{\left(x_{1}-x_{0}\right)}\right) \\
& =y_{0}\left(x_{1}-x_{0}\right)-\frac{y_{0}}{2}\left(x_{1}-x_{0}\right)+\frac{y_{1}}{2}\left(x_{1}-x_{0}\right) \\
& =\frac{\left(x_{1}-x_{0}\right)}{2}\left(2 y_{0}-y_{0}+y_{1}\right)=\frac{\left(x_{1}-x_{0}\right)}{2}\left(y_{0}+y_{1}\right)
\end{aligned}
$$

$$
\int_{x_{1}}^{x_{2}} y d x=\frac{\left(x_{2}-x_{1}\right)}{2}\left(y_{1}+y_{2}\right)
$$

Similarly, for the interval $\left[x_{2}, x_{3}\right]$ we deduce,

Similarly for the interval $\left[x_{i}, x_{i+1}\right]$ we have

$$
\int_{x_{2}}^{x_{3}} y d x=\frac{\left(x_{3}-x_{2}\right)}{2}\left(y_{2}+y_{3}\right)
$$

Now from (1) we get

$$
\int_{x_{i}}^{x_{i+1}} y d x=\frac{\left(x_{i+1}-x_{i}\right)}{2}\left(y_{i}+y_{i+1}\right)
$$

$$
\begin{gathered}
I=\frac{\left(x_{i+1}-x_{i}\right)}{2} \int_{y_{j}}^{y_{j+1}}\left[f\left(x_{i}, y\right)+f\left(x_{i+1}, y\right)\right] d y \\
=\frac{\left(x_{i+1}-x_{i}\right)}{2} \frac{\left(y_{j+1}-y_{j}\right)}{2}\left[f\left(x_{i}, y_{j}\right)+f\left(x_{i}, y_{j+1}\right)+f\left(x_{i+1}, y_{j}\right)+f\left(x_{i+1}, y_{j+1}\right)\right] \\
=\frac{\left(x_{i+1}-x_{i}\right)\left(y_{j+1}-y_{j}\right)}{4}\left[f\left(x_{i}, y_{j}\right)+f\left(x_{i+1}, y_{j}\right)+f\left(x_{i}, y_{j+1}\right)+f\left(x_{i+1}, y_{j+1}\right)\right] \\
=\frac{\left(x_{i+1}-x_{i}\right)\left(y_{j+1}-y_{j}\right)}{4}\left[f_{i, j}+f_{i+1, j}+f_{i, j+1}+f_{i+1, j+1}\right]
\end{gathered}
$$

Where, $f_{i, j}=f\left(x_{i}, y_{j}\right)$$$
\text { This is the general formula of double integral for uneven space. }
$$

\section{Numerical Examples}

In this chapter, we will discuss some numerical examples and using numerical integration formula for unequal data space and we try to find their solutions. Then we compared numerical result with the exact solution to determine corresponding errors and check out which methods provide better results. 


\subsection{Investigation by First Example}

Consider a rectangular double integral,

$$
f(x, y)=\int_{0}^{2} \int_{0}^{3} e^{y-x} d y d x
$$

We divide each of the intervals [0, 2] and [0, 3] into 60 subintervals

Table 1 . Different values of $x$ and $y$ in interval $[0,2]$ and $[0,3]$ respectively

\begin{tabular}{|c|c|c|c|c|c|}
\hline $\begin{array}{l}\text { No. of } \\
\text { Points }\end{array}$ & Points of $\mathrm{x}$ & Points of $y$ & No. of Points & Points of $\mathrm{x}$ & Points of $y$ \\
\hline 1 & 0 & 0 & 32 & 1.06 & 1.55 \\
\hline 2 & 0.10 & 0.15 & 33 & 1.10 & 1.65 \\
\hline 3 & 0.13 & 0.20 & 34 & 1.12 & 1.70 \\
\hline 4 & 0.15 & 0.22 & 35 & 1.15 & 1.75 \\
\hline 5 & 0.17 & 0.25 & 36 & 1.18 & 1.80 \\
\hline 6 & 0.20 & 0.30 & 37 & 1.24 & 1.84 \\
\hline 7 & 0.24 & 0.37 & 38 & 1.28 & 1.90 \\
\hline 8 & 0.28 & 0.42 & 39 & 1.30 & 1.95 \\
\hline 9 & 0.30 & 0.45 & 40 & 1.33 & 2.0 \\
\hline 10 & 0.33 & 0.50 & 41 & 1.37 & 2.10 \\
\hline 11 & 0.37 & 0.56 & 42 & 1.40 & 2.15 \\
\hline 12 & 0.39 & 0.60 & 43 & 1.45 & 2.19 \\
\hline 13 & 0.42 & 0.68 & 44 & 1.50 & 2.25 \\
\hline 14 & 0.45 & 0.70 & 45 & 1.53 & 2.30 \\
\hline 15 & 0.50 & 0.75 & 46 & 1.57 & 2.36 \\
\hline 16 & 0.53 & 0.78 & 47 & 1.60 & 2.40 \\
\hline 17 & 0.57 & 0.80 & 48 & 1.64 & 2.45 \\
\hline 18 & 0.60 & 0.87 & 49 & 1.67 & 2.50 \\
\hline 19 & 0.65 & 0.95 & 50 & 1.69 & 2.53 \\
\hline 20 & 0.67 & 1.0 & 51 & 1.72 & 2.56 \\
\hline 21 & 0.70 & 1.05 & 52 & 1.74 & 2.65 \\
\hline 22 & 0.74 & 1.10 & 53 & 1.76 & 2.70 \\
\hline 23 & 0.76 & 1.18 & 54 & 1.78 & 2.75 \\
\hline 24 & 0.78 & 1.20 & 55 & 1.80 & 2.80 \\
\hline 25 & 0.80 & 1.25 & 56 & 1.84 & 2.84 \\
\hline 26 & 0.82 & 1.30 & 57 & 1.88 & 2.90 \\
\hline 27 & 0.85 & 1.34 & 58 & 1.90 & 2.93 \\
\hline 28 & 0.90 & 1.40 & 59 & 1.92 & 2.95 \\
\hline 29 & 0.93 & 1.44 & 60 & 1.95 & 2.97 \\
\hline 30 & 0.97 & 1.47 & 61 & 2.0 & 3.0 \\
\hline 31 & 1.0 & 1.50 & & & \\
\hline
\end{tabular}

Table-1 represents the different unequal values of $\mathrm{x}$ and $\mathrm{y}$ in interval $[0,2]$ and $[0,3]$ respectively.

Now, we evaluate the integral value by using our proposed formula for unequal data points and make a comparison with exact result.

Table 2. Comparison of actual and numerical result

\begin{tabular}{|c|c|c|}
\hline Analytical Result & Numerical Result & Error \\
\hline 16.5026 & 16.5105 & 0.0079 \\
\hline
\end{tabular}

Table 2 describes the actual and numerical result and also the deviation between them and we have found that numerical result is very close to actual result.

\subsection{Investigation by Second Example}

Consider a rectangular double integral,

$$
f(x, y)=\int_{1}^{4} \int_{1}^{3} \ln (x+2 y) d y d x
$$

We divide each of the intervals [1, 4] and [1, 3] into 60 subintervals 
Table 3. Different values of $x$ and $y$ in interval $[1,4]$ and $[1,3]$ respectively

\begin{tabular}{|c|c|c|c|c|c|}
\hline $\begin{array}{l}\text { No. of } \\
\text { Points }\end{array}$ & Points of $x$ & Points of $y$ & $\begin{array}{l}\text { No. of } \\
\text { Points }\end{array}$ & Points of $x$ & Points of $y$ \\
\hline 1 & 1.0 & 1.0 & 32 & 2.55 & 2.06 \\
\hline 2 & 1.15 & 1.10 & 33 & 2.65 & 2.10 \\
\hline 3 & 1.20 & 1.13 & 34 & 2.70 & 2.12 \\
\hline 4 & 1.22 & 1.15 & 35 & 2.75 & 2.15 \\
\hline 5 & 1.25 & 1.17 & 36 & 2.80 & 2.18 \\
\hline 6 & 1.30 & 1.20 & 37 & 2.84 & 2.24 \\
\hline 7 & 1.37 & 1.24 & 38 & 2.90 & 2.28 \\
\hline 8 & 1.42 & 1.28 & 39 & 2.95 & 2.30 \\
\hline 9 & 1.45 & 1.30 & 40 & 3.0 & 2.33 \\
\hline 10 & 1.50 & 1.33 & 41 & 3.10 & 2.37 \\
\hline 11 & 1.56 & 1.37 & 42 & 3.15 & 2.40 \\
\hline 12 & 1.60 & 1.39 & 43 & 3.19 & 2.45 \\
\hline 13 & 1.68 & 1.42 & 44 & 3.25 & 2.50 \\
\hline 14 & 1.70 & 1.45 & 45 & 3.30 & 2.53 \\
\hline 15 & 1.75 & 1.50 & 46 & 3.36 & 2.57 \\
\hline 16 & 1.78 & 1.53 & 47 & 3.40 & 2.60 \\
\hline 17 & 1.80 & 1.57 & 48 & 3.45 & 2.64 \\
\hline 18 & 1.87 & 1.60 & 49 & 3.50 & 2.67 \\
\hline 19 & 1.95 & 1.65 & 50 & 3.53 & 2.69 \\
\hline 20 & 2.0 & 1.67 & 51 & 3.56 & 2.72 \\
\hline 21 & 2.05 & 1.70 & 52 & 3.60 & 2.74 \\
\hline 22 & 2.10 & 1.74 & 53 & 3.65 & 2.76 \\
\hline 23 & 2.18 & 1.76 & 54 & 3.70 & 2.78 \\
\hline 24 & 2.20 & 1.78 & 55 & 3.75 & 2.80 \\
\hline 25 & 2.25 & 1.80 & 56 & 3.80 & 2.84 \\
\hline 26 & 2.30 & 1.82 & 57 & 3.84 & 2.88 \\
\hline 27 & 2.34 & 1.85 & 58 & 3.90 & 2.90 \\
\hline 28 & 2.40 & 1.90 & 59 & 3.93 & 2.92 \\
\hline 29 & 2.44 & 1.93 & 60 & 3.95 & 2.95 \\
\hline 30 & 2.47 & 1.97 & 61 & 4.0 & 3.0 \\
\hline 31 & 2.50 & 2.0 & & & \\
\hline
\end{tabular}

Table-3 represents the different unequal values of $\mathrm{x}$ and $\mathrm{y}$ in interval [1, 4] and [1, 3] respectively.

Now, we evaluate the integral value by using our proposed formula for unequal data points and make a comparison with exact result.

Table 4. Comparison of actual and numerical result

\begin{tabular}{|c|c|c|}
\hline Analytical Result & Numerical Result & Error \\
\hline 11.0733 & 11.0731 & 0.0002 \\
\hline
\end{tabular}

Table 4 describes the actual and numerical result and also the deviation between them and we have found that numerical result is very close to analytical result.

From the above two investigation, we found that our generated mathematical formula is convenient to solve numerical double integration problems when the data values are not equal.

\section{Conclusion}

Numerical Double integrals are widely used to calculate the area of a region and the volume under a surface when the analytical result cannot be found easily. It is also difficult to find numerical solution when the data values are not equal. In this work we generate a numerical double integration formula for unequal data space. For that, Trapezoidal rule for unequal data space have been used to evaluate double integration formula. We also applied our mathematical model by investigating some numerical examples and we have found a feasible result. Our research will be helpful in many scientific areas where numerical solution is needed.

\section{References}

[1] M. Buchibrand, 7 forms of divorce in Africa, Retrieved on 22nd May 2018 from https://www.nairaland.com/2526539/7-formsdivorce-africa, 2016. [1] Al-Jarrah, R. On the Lagrange Interpolation Polynomials. Journal of Approximation Theory 41, 170-1 78, 1884.

[2] Anton, H., Bivens, I., \& Davis, S. Calculas Early Transcendentals. United States of America: John Wiley \& Sons, Inc, 2012. 
[3] Das, B., \& Chakrabarty, D. Lagrange’s Interpolation Formula: Representation of Numerical Data by a Polynomial curve. International Journal of Mathematics Trends and Technology (IJMTT), 3, 2016.

[4] Dahiya, V. Analysis of Lagrange Interpolation Formula. IJISET - International Journal of Innovative Science, Engineering \& Technology, 1(10), 2014.

[5] Darkwah, K. A., Norttey, E. N. N., \& Anan, C. A Proposed Numerical Integration Method Using Polynomial Interpolation. British Journal of Mathematics \& Computer Science, 16, 1-10, 2016.

[6] Dhali, M. N., Bulbul, M. F., \& Sadiya, U. Comparison on Trapezoidal and Simpson's Rule for Unequal Data Space. International Journal of Mathematical Sciences and Computing, 5(4), 33-43, DOI: 10.5815/ijmsc.2019.04.04, 2019.

[7] Douglas, F. J., \& Burden, L. R. Numerical Analysis, Thomson Learning, 2001.

[8] Gill, P. E., \& Miller, G. F. An algorithm for the integration of unequally spaced data. Computer Journal, 15, 80-83, 1972.

[9] Hildebrand, F. B. \& Graw-Hill, M. Introduction to Numerical Analysis. New Work, 1974.

[10] Jayakumar, J. Generalized Simpson-Newton's Method for Solvinf Nonlinear Equations with Cubic Convergence. IQSR Journal of Mathematics, 7(5), 58-61, 2013.

[11] Khan, M.-U.-R., Hossain, M., \& Parvin, S. Numerical Integration Schemes for Unequal Data Spacing. American Journal of Applied Mathematics, 5(2), 48-56, 2017.

[12] Muthumalai, R. K. Some Formulas For Numerical Differentiation Through Divided Differences. International Journal of Mathematical Archive(3), 2012.

[13] Muosa, S. M. Numerical Methods for Evaluation of Double integrals with Continuous Integrands, International Journal of Pure and Applied Mathematics, Volume 119, No. 10, 385-396, 2018.

[14] Saputra, A., Bakri, R. and Mahmud, R. Numerical Analysis of Double Integral of Trigonometric Function Using Romberg Method. Daya Matematis: Jurnal Inovasi Pendidikan Matematika, Vol. 2, No. 2, 131-136, 2020.

[15] Sinha, R K and Kumar, R. Numerical method for evaluating the integrable function on a finite interval. International Journal of Engineering Science and Technology. 2(6), 2010.

[16] Sozio, G. Numerical Integration. Australian Senior Mathematics Journal. 23(1), 2009.

[17] Thukral, R. Further Acceleration of the Simpson's Method for Solving Non-linear Equation. Journal of Advances in Mathematics, 14, 02, 2018.

\section{Authors' Profiles}

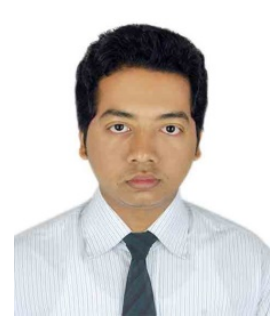

Md. Nayan Dhali was born in Bangladesh. He has completed B S (Honor's) and M S in Mathematics from University of Dhaka. Now he is performing as an Assistant Professor of Mathematics at Department of Mathematics in Jashore University of Science and Technology, Bangladesh. Email: mnd_math@just.edu.bd

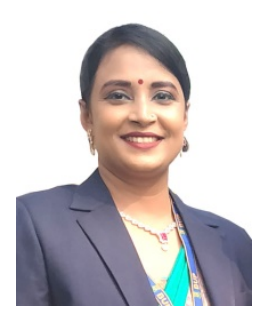

Nandita Barman was born in Bangladesh. She has completed B S (Honor's) and M S in Mathematics from University of Dhaka. Now she is performing as an Assistant Professor of Mathematics at Department of ICT in Bangladesh University of Professionals, Bangladesh. Email: nanditabarmandu12@gmail.com

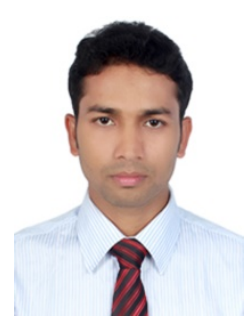

Md. Mohedul Hasan was born in Bangladesh. He has completed B S (Honor's) and M S in Mathematics from University of Dhaka. Now he is performing as a Lecturer of Mathematics at Department of Mathematics in Dhaka University of Engineering and Technology, Bangladesh. mohedul.math@gmail.com 


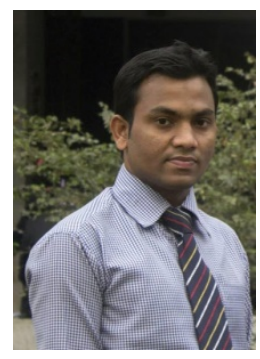

A. K. M. Selim Reza was born in Bangladesh. He has completed B S (Honor's) and M S in Mathematics from University of Dhaka. Now he is performing as a Lecturer of Mathematics at Department of Mathematics in Khulna University of Engineering and Technology, Bangladesh. Email: selim_1992@yahoo.com

How to cite this paper: Md. Nayan Dhali, Nandita Barman, Md. Mohedul Hasan, A. K. M. Selim Reza. " Numerical Double Integration for Unequal Data Spaces ", International Journal of Mathematical Sciences and Computing (IJMSC), Vol.6, No.6, pp.2429, 2020. DOI: 10.5815/IJMSC.2020.06.04 